\title{
Impulsive Control Strategy for a Nonautonomous Food-Chain System with Multiple Delays
}

\author{
Baodan Tian, Yanhong Qiu, and Yucai Ding
}

Institute of Modeling and Algorithm, School of Science, Southwest University of Science and Technology, Mianyang 621010, China

Correspondence should be addressed to Baodan Tian; tianbaodan@swust.edu.cn

Received 26 January 2016; Revised 8 October 2016; Accepted 19 October 2016

Academic Editor: Petko Petkov

Copyright (c) 2016 Baodan Tian et al. This is an open access article distributed under the Creative Commons Attribution License, which permits unrestricted use, distribution, and reproduction in any medium, provided the original work is properly cited.

\begin{abstract}
A nonautonomous food-chain system with Holling II functional response is studied, in which multiple delays of digestion are also considered. By applying techniques in differential inequalities, comparison theorem in ordinary differential equations, impulsive differential equations, and functional differential equations, some effective control strategies are obtained for the permanence of the system. Furthermore, effects of some important coefficients and delays on the permanence of the system are intuitively and clearly shown by series of numerical examples.
\end{abstract}

\section{Introduction}

From the beginning of the early 21st century, people began to study the complexity of biology by using a variety of mathematical models and methods; then the development of mathematical biology entered a new period, especially in the field of population dynamics (see [1]) and epidemic dynamics. Moreover, some emerging theoretical tools such as the complex network were also introduced to study mathematical models in biology (see [2-4]) and so forth.

Meanwhile, it is known that many evolution processes are characterized by the fact at certain moments when they experience a change of state abruptly. These processes are subject to short-term perturbations whose duration is negligible in comparison with the duration of the process. Thus, models involving impulsive effects seem to be a hot research field (see [5-8]), which could describe the real relationship among the species more accurately. On the other hand, when prey-predator system is referred, sometimes there is a digest and absorption time (which is always called the digest delay) during the predation instead of transforming the food into growth rate immediately. Hence, in order to model the relationship between the predator and the prey more accurately, it is more reasonable to introduce time delay into the model.
Enlightened by above ecological backgrounds and based on model (1.1) in [5], we consider a nonautonomous threespecies food-chain system with multiple delays and impulsive perturbation in this paper, and the model is described as the following impulsive differential equations:

$$
\begin{aligned}
& \frac{d x}{d t}=r_{1}(t) x(t)\left(1-\frac{x\left(t-\tau_{1}\right)}{k_{1}(t)}\right)-\frac{a_{1}(t) x(t) y(t)}{b_{1}(t)+x(t)} \\
& \quad-\frac{a_{2}(t) x(t) z(t)}{b_{2}(t)+x(t)}, \\
& \frac{d y}{d t}=r_{2}(t) y(t)\left(1-\frac{y\left(t-\tau_{2}\right)}{k_{2}(t)}\right) \\
& \quad+\frac{e_{1}(t) a_{1}(t) x\left(t-\tau_{4}\right) y(t)}{b_{1}(t)+x\left(t-\tau_{4}\right)}-\frac{a_{3}(t) y(t) z(t)}{b_{3}(t)+y(t)}, \\
& \frac{d z}{d t}=z(t)\left(\frac{e_{2}(t) a_{2}(t) x\left(t-\tau_{5}\right)}{b_{2}(t)+x\left(t-\tau_{5}\right)}\right. \\
& \left.\quad+\frac{e_{3}(t) a_{3}(t) y\left(t-\tau_{6}\right)}{b_{3}(t)+y\left(t-\tau_{6}\right)}-m(t) z\left(t-\tau_{3}\right)\right), \\
& x\left(t^{+}\right)=\left(1+q_{1 k}\right) x(t),
\end{aligned}
$$




$$
\begin{aligned}
& y\left(t^{+}\right)=\left(1+q_{2 k}\right) y(t), \\
& z\left(t^{+}\right)=\left(1+q_{3 k}\right) z(t),
\end{aligned}
$$$$
t=t_{k}, k \in N^{*}
$$

where $x(t), y(t)$, and $z(t)$ denote the population of the prey and the lower and higher predator at time $t$, respectively. $r_{1}(t)$ and $r_{2}(t)$ are the intrinsic growth rate of the prey $x(t)$ and the lower predator $y(t) ; k_{1}(t)$ and $k_{2}(t)$ denote the environmental carrying capacity of the prey and the lower predator. $a_{i}(t)$ and $b_{i}(t)$ are the coefficient of the functional response, $m(t)$ is the density dependent coefficient of the higher predator $z(t)$, and $e_{i}(t)(i=1,2,3)$ represents the transform coefficients during the predation. More details of the background of this model can be found in [5].

Let $I=\left\{\left\{t_{k}\right\} \in R: t_{k}<t_{k+1}, k \in N^{*}, \lim _{k \rightarrow \pm \infty} t_{k}=\right.$ $\pm \infty\}$, and the set of all sequences are bounded and strictly increasing. Let $\Omega \subset R, \Omega \neq \varnothing, \tau=\max _{1 \leq i \leq 6}\left\{\tau_{i}\right\}, \xi_{0} \in R$. $\operatorname{PC}\left(\xi_{0}\right)$ is the space of all function $\phi:\left[\xi_{0}-\tau, \xi_{0}\right] \rightarrow \Omega$ having points of discontinuity at $\mu_{1}, \mu_{2}, \ldots, \in\left[\xi_{0}-\tau, \xi_{0}\right]$ of the first kind and left continuous at these points.

For $J \subset R, \operatorname{PC}(J, R)$ is the space of all piecewise continuous functions from $J$ to $R$ with points of discontinuity of the first kind $t_{k}$ at which it is left continuous.

Let $\phi_{i} \in \mathrm{PC}(0)(i=1,2,3)$, denote $x(t)=x\left(t ; 0, \phi_{1}\right)$, $y(t)=y\left(t ; 0, \phi_{2}\right), z(t)=z\left(t ; 0, \phi_{3}\right)$, and $(x(t), y(t), z(t))^{T}$ is the solution of system (1) satisfying the following initial conditions:

$$
\begin{aligned}
0 \leq x\left(t ; 0, \phi_{1}\right) & =\phi_{1}(s) \leq \infty \\
s & \in[-\tau, 0], x\left(0+0 ; 0, \phi_{1}\right)=\phi_{1}(0)>0 \\
0 \leq y\left(t ; 0, \phi_{2}\right) & =\phi_{2}(s) \leq \infty \\
s & \in[-\tau, 0], y\left(0+0 ; 0, \phi_{2}\right)=\phi_{2}(0)>0 ; \\
0 \leq z\left(t ; 0, \phi_{3}\right) & =\phi_{3}(s) \leq \infty, \\
s & \in[-\tau, 0], z\left(0+0 ; 0, \phi_{3}\right)=\phi_{3}(0)>0 .
\end{aligned}
$$

It is easy to verify that solutions of system (1) with above initial conditions are positive for all $t \geq 0$.

Furthermore, throughout the present paper, we assume

(H1) $0<t_{1}<t_{2} \cdots<t_{k}<\cdots$ are fixed impulsive points with $\lim _{k \rightarrow \infty} t_{k}=\infty$;

(H2) $q_{i k}$ are real sequences with $q_{i k}>-1$;

(H3) there exist some positive constants $M_{i}$ and $m_{i}$ such that

$$
m_{i} \leq \prod_{0<t_{k}<t}\left(1+q_{i k}\right) \leq M_{i}, \quad i=1,2,3
$$

where $k \in N^{*} ; N^{*}$ denotes set of the positive integer.
For a real positive and continuous function $f(t)$, we denote

$$
\begin{aligned}
f^{M} & =\max _{t \in[0, \infty)} f(t), \\
f^{L} & =\min _{t \in[0, \infty)} f(t) .
\end{aligned}
$$

\section{Preliminaries}

In this section, we will consider the following impulsive system (5) with time delay and give two important lemmas:

$$
\begin{gathered}
u^{\prime}(t)=u(t)(A-B u(t-\tau)), \quad t \neq t_{k}, \\
u\left(t^{+}\right)=\left(1+q_{i k}\right) u(t), \quad t=t_{k}, \quad k \in N^{*} .
\end{gathered}
$$

Under the hypotheses (H1)-(H3), the corresponding nonimpulsive system of system reads

$$
v^{\prime}(t)=v(t)\left(A-B \prod_{0<t_{k}<t}\left(1+q_{i k}\right) v(t-\tau)\right) .
$$

Lemma 1 (see [9]). Assume that (H1)-(H3) hold:

(1) If $v(t)$ is a solution of system (6) on $[-\tau, \infty)$, then

$$
u(t)=\prod_{0<t_{k}<t}\left(1+q_{i k}\right) v(t)
$$

is a solution of system (5) on $[-\tau, \infty)$.

(2) If $u(t)$ is a solution of system (5) on $[-\tau, \infty)$, then

$$
v(t)=\prod_{0<t_{k}<t}\left(1+q_{i k}\right)^{-1} u(t)
$$

is a solution of system (6) on $[-\tau, \infty)$.

The proof of this lemma can be found in [10], and we omit it here.

In the following, we will give the other important lemma, which was introduced by Gopalsamy in [11] (page 57: Theorem 1.4.3).

For a delay logistic equation of the form

$$
N^{\prime}(t)=N(t)\left(b-a_{1} N\left(t-\tau_{1}\right)-a_{2} N\left(t-\tau_{2}\right)\right),
$$

with initial conditions,

$$
\begin{aligned}
& N(s)=\varphi(s) \geq 0, \\
& s \in[-\tau, 0], \tau=\max \left\{\tau_{1}, \tau_{2}\right\}, \varphi \in C\left([-\tau, 0], R_{+}\right), \varphi(0)>0,
\end{aligned}
$$

where $b, a_{1}, a_{2}, \tau_{1}, \tau_{2} \in(0,+\infty)$.

Lemma 2. For the delay logistic equation (9) with initial conditions (10), if condition

$$
b\left(\tau_{1}+\tau_{2}\right) \exp \left[b\left(\tau_{1}+\tau_{2}\right)\right]<1
$$

holds, then

$$
\lim _{t \rightarrow \infty} N(t)=\frac{b}{a_{1}+a_{2}} .
$$


By the above lemma, we have the following corollary.

Corollary 3. For any solution $u(t)$ of the functional differential equation

$$
u^{\prime}(t)=u(t)(A-B u(t-\tau)),
$$

with initial condition

$$
u(s)=\varphi(s) \in C\left([-\tau, 0], R^{+}\right), \quad \varphi(0)>0,
$$

if condition

(H4)

$$
A \tau e^{A \tau}<1
$$

holds, then

$$
\lim _{t \rightarrow \infty} u(t)=\frac{A}{B}
$$

\section{Main Results}

Theorem 4. Assume that (H1)-(H4) hold; then for any solution of system (1) there exist $T_{0}>0$ and $\eta_{i}>0$ such that $x(t) \leq \eta_{1}, y(t) \leq \eta_{2}, z(t) \leq \eta_{3}$ for $t>T_{0}$.

Proof. From the first equation of system (1), we have

$$
\begin{aligned}
& x^{\prime}(t) \leq r_{1}^{M} x(t)\left(1-\frac{x\left(t-\tau_{1}\right)}{k_{1}^{M}}\right), \quad t \neq t_{k}, \\
& x\left(t^{+}\right)=\left(1+q_{1 k}\right) x(t), \quad t=t_{k}, k \in N^{*},
\end{aligned}
$$

whose corresponding comparison impulsive system is

$$
\begin{aligned}
& u_{1}^{\prime}(t)=r_{1}^{M} u_{1}(t)\left(1-\frac{u_{1}\left(t-\tau_{1}\right)}{k_{1}^{M}}\right), \quad t \neq t_{k}, \\
& u_{1}\left(t^{+}\right)=\left(1+q_{1 k}\right) u_{1}(t), \quad t=t_{k}, k \in N^{*} .
\end{aligned}
$$

According to Lemma 1, the corresponding nonimpulsive equation of (18) reads

$$
\begin{aligned}
& v_{1}^{\prime}(t) \\
& \quad=r_{1}^{M} v_{1}(t)\left(1-\frac{1}{k_{1}^{M}} \prod_{0<t_{k}<t}\left(1+q_{1 k}\right) v_{1}\left(t-\tau_{1}\right)\right) .
\end{aligned}
$$

By hypothesis (H3), it follows from (19) that

$$
v_{1}^{\prime}(t) \leq r_{1}^{M} v_{1}(t)\left(1-\frac{m_{1}}{k_{1}^{M}} v_{1}\left(t-\tau_{1}\right)\right),
$$

whose corresponding comparison functional differential system is

$$
w_{1}^{\prime}(t)=r_{1}^{M} w_{1}(t)\left(1-\frac{m_{1}}{k_{1}^{M}} w_{1}\left(t-\tau_{1}\right)\right) .
$$

By the corollary, it follows from (21) that

$$
\lim _{t \rightarrow \infty} w_{1}(t)=\frac{k_{1}^{M}}{m_{1}}=\Delta_{1} ;
$$

then, for any small positive $\varepsilon_{1}>0$, there exists $T_{1}>0$, such that $w_{1}(t)<\Delta_{1}+\varepsilon_{1}$ for $t>T_{1}$.

On the other hand, by hypothesis ( $\mathrm{H} 3)$, the comparison theorem on impulsive differential equation (see [12]), and the comparison theorem on functional differential equation (see [13]), we have

$$
\begin{aligned}
x(t) & \leq u_{1}(t)=\prod_{0<t_{k}<t}\left(1+q_{1 k}\right) v_{1}(t) \leq M_{1} v_{1}(t) \\
& \leq M_{1} w_{1}(t) \leq M_{1}\left(\Delta_{1}+\varepsilon_{1}\right)=\eta_{1} .
\end{aligned}
$$

Also, from the second and the third equations of system (1), when $t \geq T_{1}$ we have

$$
\begin{aligned}
& y^{\prime}(t) \leq y(t)\left(r_{2}^{M}+\frac{e_{1}^{M} a_{1}^{M} \eta_{1}}{b_{1}^{L}+\eta_{1}}-\frac{r_{2}^{M} y\left(t-\tau_{2}\right)}{k_{2}^{M}}\right), \\
& y\left(t^{+}\right)=\left(1+q_{2 k}\right) y(t), \quad t=t_{k}, k \in N^{*},
\end{aligned}
$$

whose comparison system is

$$
\begin{array}{r}
u_{2}^{\prime}(t)=u_{2}(t)\left(r_{2}^{M}+\frac{e_{1}^{M} a_{1}^{M} \eta_{1}}{b_{1}^{L}+\eta_{1}}-\frac{r_{2}^{M} u_{2}\left(t-\tau_{2}\right)}{k_{2}^{M}}\right), \\
t \neq t_{k},
\end{array}
$$

$u_{2}\left(t^{+}\right)=\left(1+q_{2 k}\right) u_{2}(t), \quad t=t_{k}, k \in N^{*}$.

And the corresponding nonimpulsive system of (25) is

$$
\begin{aligned}
& v_{2}^{\prime}(t)=v_{2}(t)\left(r_{2}^{M}+\frac{e_{1}^{M} a_{1}^{M} \eta_{1}}{b_{1}^{L}+\eta_{1}}\right. \\
& \left.-\frac{r_{2}^{M}}{k_{2}^{M}} \prod_{0<t_{k}<t}\left(1+q_{2 k}\right) v_{2}\left(t-\tau_{2}\right)\right) \leq v_{2}(t)\left(r_{2}^{M}\right. \\
& \left.+\frac{e_{1}^{M} a_{1}^{M} \eta_{1}}{b_{1}^{L}+\eta_{1}}-\frac{m_{2} r_{2}^{M}}{k_{2}^{M}} v_{2}\left(t-\tau_{2}\right)\right)
\end{aligned}
$$

whose comparison system is

$$
\begin{aligned}
& w_{2}^{\prime}(t) \\
& \quad=w_{2}(t)\left(r_{2}^{M}+\frac{e_{1}^{M} a_{1}^{M} \eta_{1}}{b_{1}^{L}+\eta_{1}}-\frac{m_{2} r_{2}^{M}}{k_{2}^{M}} w_{2}\left(t-\tau_{2}\right)\right) .
\end{aligned}
$$

By the corollary again, it follows from (27) that

$$
\lim _{t \rightarrow \infty} w_{2}(t)=\frac{r_{2}^{M}+e_{1}^{M} a_{1}^{M} \eta_{1} /\left(b_{1}^{L}+\eta_{1}\right)}{m_{2} r_{2}^{M} / k_{2}^{M}}=\Delta_{2} ;
$$



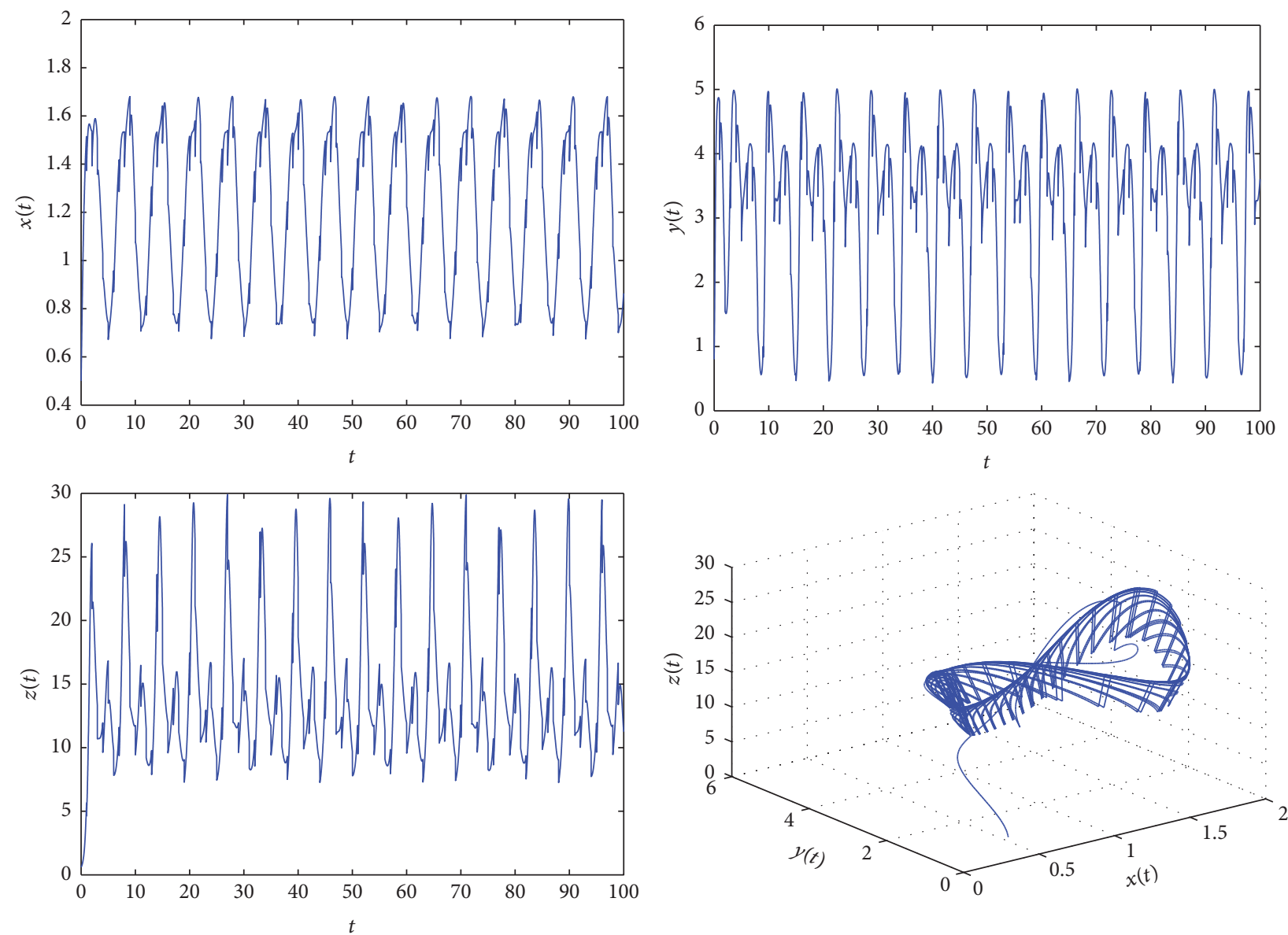

FIGURE 1: Permanence of system (1) with the impulsive control strategy with $\phi_{1}(s)=x(0)=0.5, \phi_{2}(s)=y(0)=0.8, \phi_{3}(s)=z(0)=0.6, s \in$ $[-\tau, 0]$.

then, for any small positive $\varepsilon_{2}>0$, there exists $T_{2}>0$, such that $w_{2}(t)<\Delta_{2}+\varepsilon_{2}$ for $t>T_{2}$.

Thus, by hypothesis (H3) and the comparison theorem we have

$$
\begin{aligned}
y(t) & \leq u_{2}(t)=\prod_{0<t_{k}<t}\left(1+q_{2 k}\right) v_{2}(t) \leq M_{2} v_{2}(t) \\
& \leq M_{2} w_{2}(t) \leq M_{2}\left(\Delta_{2}+\varepsilon_{2}\right)=\eta_{2} .
\end{aligned}
$$

Finally, when $t \geq T_{2}$ it follows from the third equation of system (1) that we have

$$
\begin{array}{r}
z^{\prime}(t) \leq z(t)\left(\frac{e_{2}^{M} a_{2}^{M} \eta_{1}}{b_{2}^{L}+\eta_{1}}+\frac{e_{3}^{M} a_{3}^{M} \eta_{2}}{b_{3}^{L}+\eta_{2}}-m^{L} z\left(t-\tau_{3}\right)\right) \\
t \neq t_{k},
\end{array}
$$

$z\left(t^{+}\right)=\left(1+q_{3 k}\right) z(t), \quad t=t_{k}, k \in N^{*}$.

Repeating the above process, we can derive that, for any small positive $\varepsilon_{2}>0$, there exists $T_{0}>0$, such that

$$
z(t) \leq M_{3}\left(\Delta_{3}+\varepsilon_{3}\right)=\eta_{3} \text { for } t>T_{0}
$$

where

$$
\Delta_{3}=\frac{1}{m^{L} m_{3}}\left(\frac{e_{2}^{M} a_{2}^{M} \eta_{1}}{b_{2}^{L}+\eta_{1}}+\frac{e_{3}^{M} a_{3}^{M} \eta_{2}}{b_{3}^{L}+\eta_{2}}\right) .
$$

Theorem 5. If (H1)-(H4) hold, further assume that

$$
r_{1}^{L}>\frac{a_{1}^{M} \eta_{1} \eta_{2}}{b_{2}^{L}+\eta_{1}}+\frac{a_{2}^{M} \eta_{1} \eta_{3}}{b_{2}^{L}+\eta_{1}}
$$

(H6)

$$
r_{2}^{L}+\frac{a_{2}^{L} e_{2}^{L} \xi_{1}}{b_{1}^{M}+\xi_{1}}>\frac{a_{3}^{M} \eta_{3}}{\mathrm{~b}_{3}^{L}}
$$



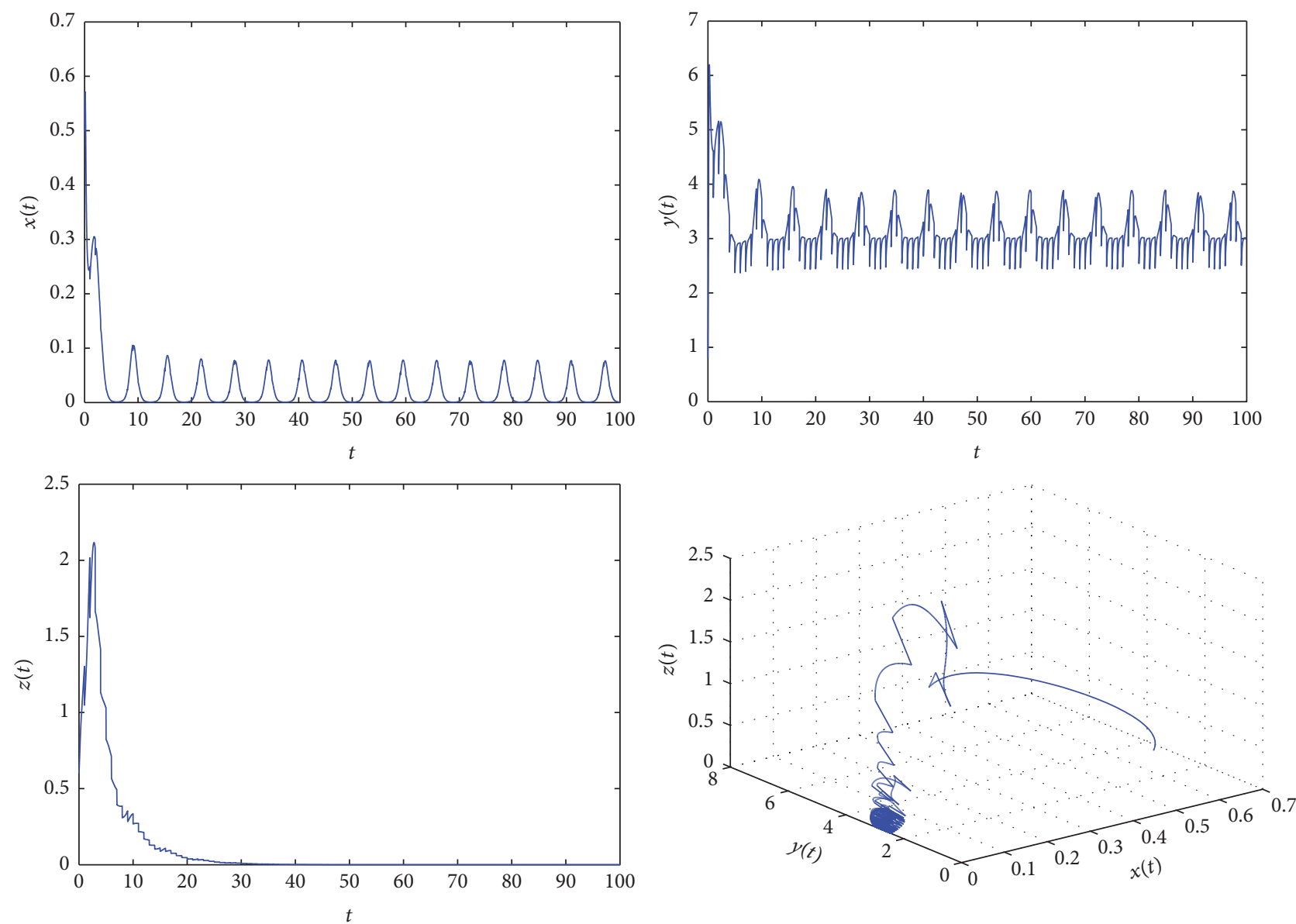

FIGURE 2: Extinction of the higher predator of system (1) with $\phi_{1}(s)=x(0)=0.5, \phi_{2}(s)=y(0)=0.8, \phi_{3}(s)=z(0)=0.6, s \in[-\tau, 0]$.

hold; then for any solution of system (1) there exist $T>T_{0}$ and $\xi_{i}>0(i=1,2,3)$ such that

$$
\begin{aligned}
& x(t) \geq \xi_{1}, \\
& y(t) \geq \xi_{2}, \\
& z(t) \geq \xi_{3},
\end{aligned}
$$

\section{for $t>T$.}

Proof. From the first equation of system (1), when $t>T_{0}$,

$x^{\prime}(t)$

$$
\begin{array}{r}
\geq x(t)\left(r_{1}^{L}-\frac{a_{1}^{M} \eta_{1} \eta_{2}}{b_{1}^{L}+\eta_{1}}-\frac{a_{2}^{M} \eta_{1} \eta_{3}}{b_{2}^{L}+\eta_{1}}-\frac{r_{1}^{M}}{k_{1}^{L}} x\left(t-\tau_{1}\right)\right), \\
t \neq t_{k},
\end{array}
$$

$x\left(t^{+}\right)=\left(1+q_{1 k}\right) x(t), \quad t=t_{k}, k \in N^{*}$, whose corresponding comparison impulsive system is

$$
\begin{aligned}
& u_{3}^{\prime}(t)=u_{3}(t) \\
& \cdot\left(r_{1}^{L}-\frac{a_{1}^{M} \eta_{1} \eta_{2}}{b_{1}^{L}+\eta_{1}}-\frac{a_{2}^{M} \eta_{1} \eta_{3}}{b_{2}^{L}+\eta_{1}}-\frac{r_{1}^{M}}{k_{1}^{L}} u_{3}\left(t-\tau_{1}\right)\right), \\
& u_{3}\left(t^{+}\right)=\left(1+q_{1 k}\right) u_{3}(t), \quad t=t_{k}, k \in N^{*},
\end{aligned}
$$

By Lemma 1, the corresponding nonimpulsive equation of system (37) reads

$$
\begin{aligned}
v_{3}^{\prime}(t) & =v_{3}(t)\left(r_{1}^{L}-\frac{a_{1}^{M} \eta_{1} \eta_{2}}{b_{1}^{L}+\eta_{1}}-\frac{a_{2}^{M} \eta_{1} \eta_{3}}{b_{2}^{L}+\eta_{1}}\right. \\
- & \left.\frac{r_{1}^{M}}{k_{1}^{L}} \prod_{0<t_{k}<t}\left(1+q_{1 k}\right) v_{3}\left(t-\tau_{1}\right)\right) \geq v_{3}(t)\left(r_{1}^{L}\right. \\
- & \left.\frac{a_{1}^{M} \eta_{1} \eta_{2}}{b_{1}^{L}+\eta_{1}}-\frac{a_{2}^{M} \eta_{1} \eta_{3}}{b_{2}^{L}+\eta_{1}}-\frac{r_{1}^{M} M_{1}}{k_{1}^{L}} v_{3}\left(t-\tau_{1}\right)\right),
\end{aligned}
$$



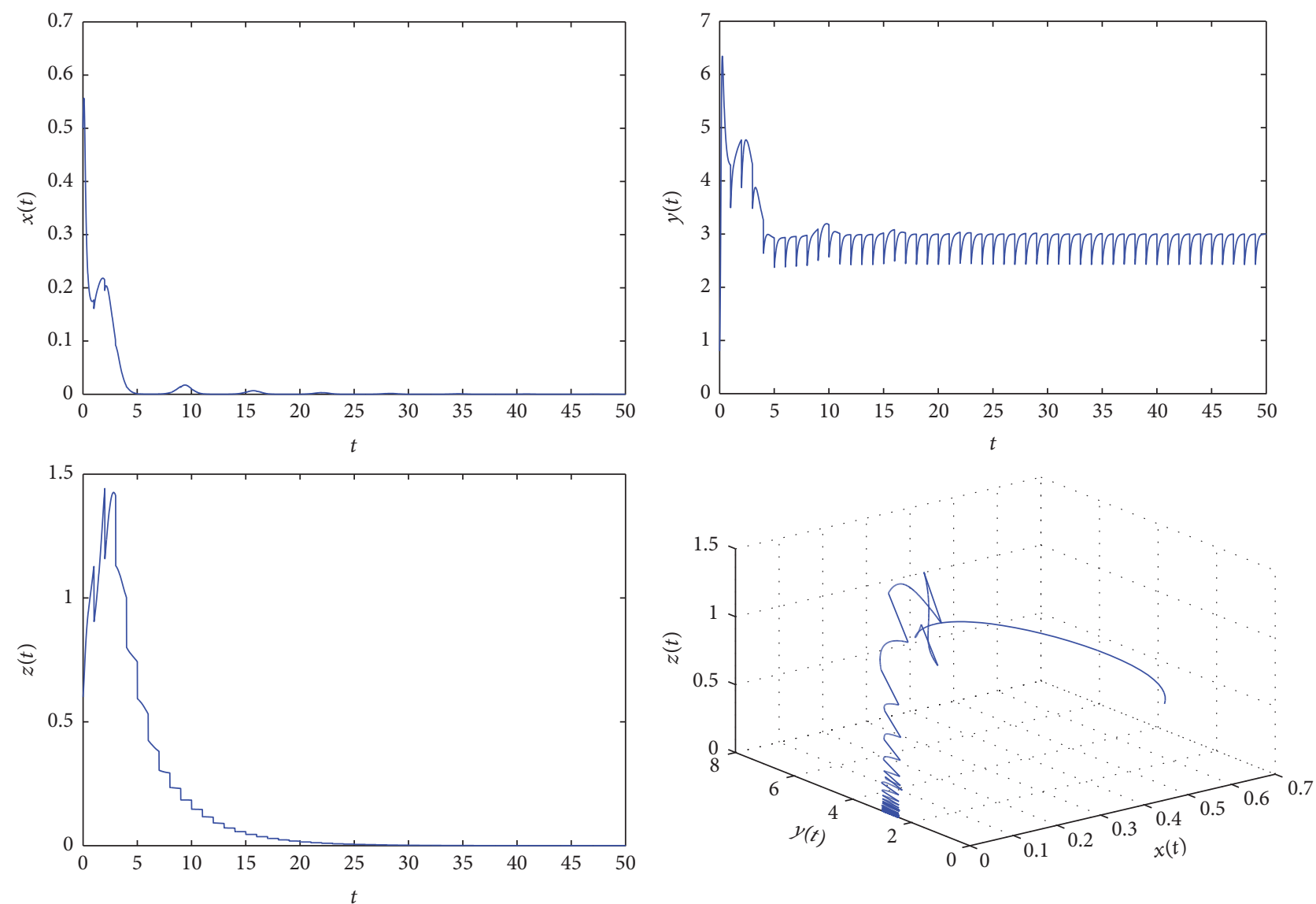

FIGURE 3: Extinction of the prey of system (1) with $\phi_{1}(s)=x(0)=0.5, \phi_{2}(s)=y(0)=0.8, \phi_{3}(s)=z(0)=0.6, s \in[-\tau, 0]$.

and the comparison equation of $(38)$ is

$$
\begin{aligned}
& w_{3}^{\prime}(t)=w_{3}(t) \\
& \quad \cdot\left(r_{1}^{L}-\frac{a_{1}^{M} \eta_{1} \eta_{2}}{b_{1}^{L}+\eta_{1}}-\frac{a_{2}^{M} \eta_{1} \eta_{3}}{b_{2}^{L}+\eta_{1}}-\frac{r_{1}^{M} M_{1}}{k_{1}^{L}} w_{3}\left(t-\tau_{1}\right)\right) .
\end{aligned}
$$

For the functional differential equation (39), it follows from the corollary that $\lim _{t \rightarrow \infty} w_{3}(t)=\delta_{1}$, where

$$
\delta_{1}=\frac{k_{1}^{L}}{r_{1}^{M} M_{1}}\left(r_{1}^{L}-\frac{a_{1}^{M} \eta_{1} \eta_{2}}{b_{1}^{L}+\eta_{1}}-\frac{a_{2}^{M} \eta_{1} \eta_{3}}{b_{2}^{L}+\eta_{1}}\right) .
$$

Then, for any small positive $\varepsilon_{4}>0$, there exists $T_{3}>T_{0}$, such that $w_{3}(t)>\delta_{1}-\varepsilon_{4}$ for $t>T_{0}$.

On the other hand, by hypothesis (H3) and the comparison theorem again we have

$$
\begin{aligned}
x(t) & \geq u_{3}(t)=\prod_{0<t_{k}<t}\left(1+q_{1 k}\right) v_{3}(t) \geq m_{1} v_{3}(t) \\
& \leq m_{1} w_{3}(t) \geq m_{1}\left(\delta_{1}-\varepsilon_{4}\right)=\xi_{1} .
\end{aligned}
$$

Consider the second equation of system (1); when $t>T_{3}$ we have

$$
\begin{aligned}
& y^{\prime}(t) \\
& \geq y(t)\left(r_{2}^{L}+\frac{a_{1}^{L} e_{1}^{L} \xi_{1}}{b_{1}^{M}+\xi_{1}}-\frac{a_{3}^{M} \eta_{3}}{b_{3}^{L}+\eta_{3}}-\frac{r_{2}^{M}}{k_{2}^{L}} y\left(t-\tau_{2}\right)\right), \\
& t \neq t_{k} \text {, } \\
& y\left(t^{+}\right)=\left(1+q_{2 k}\right) y(t), \quad t=t_{k}, k \in N^{*} .
\end{aligned}
$$

Repeating the above process, we can derive that, for any small positive $\varepsilon_{5}>0$, there exists $T_{4}>T_{3}>T_{0}$, such that

$$
y(t) \geq m_{2}\left(\delta_{2}-\varepsilon_{5}\right)=\xi_{2}
$$

for $t>T_{4}$, where

$$
\delta_{2}=\frac{k_{2}^{L}}{r_{2}^{M} M_{2}}\left(r_{2}^{L}+\frac{a_{1}^{L} e_{1}^{L} \xi_{1}}{b_{1}^{M}+\xi_{1}}-\frac{a_{3}^{M} \eta_{3}}{b_{3}^{L}+\eta_{3}}\right) .
$$



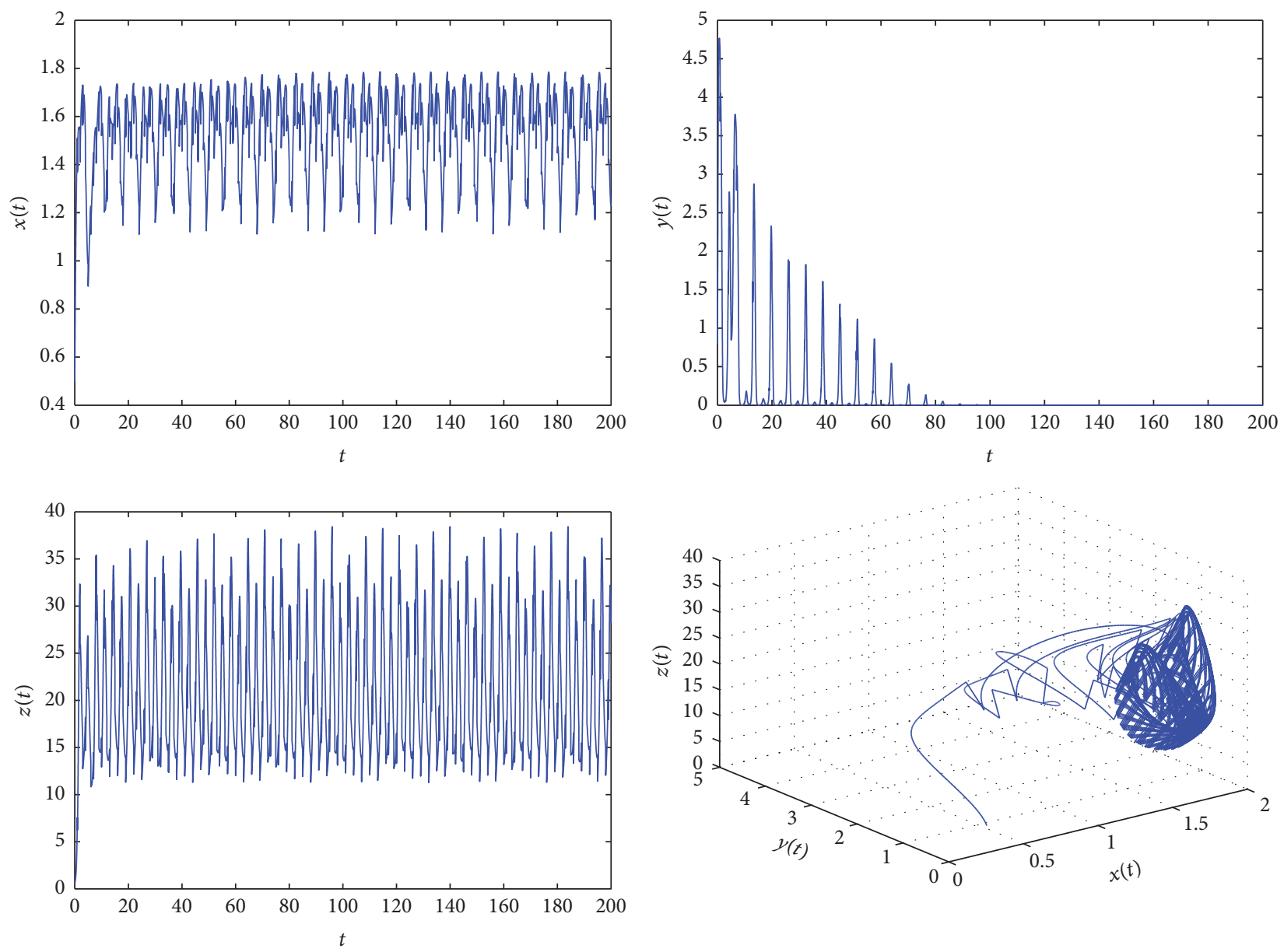

FIGURE 4: Extinction of the lower predator of system (1) with $\phi_{1}(s)=x(0)=0.5, \phi_{2}(s)=y(0)=0.8, \phi_{3}(s)=z(0)=0.6, s \in[-\tau, 0]$.

Finally, considering the third equation of system (1), when $t>T_{4}$ we have

$$
\begin{array}{r}
z^{\prime}(t) \geq z(t)\left(\frac{a_{2}^{L} e_{2}^{L} \xi_{1}}{b_{2}^{M}+\xi_{1}}+\frac{a_{3}^{L} e_{3}^{L} \xi_{2}}{b_{3}^{M}+\xi_{2}}-m^{M} z\left(t-\tau_{3}\right)\right), \\
t \neq t_{k},
\end{array}
$$

$$
z\left(t^{+}\right)=\left(1+q_{3 k}\right) z(t), \quad t=t_{k}, k \in N^{*} .
$$

In the same way, we have that, for any small positive $\varepsilon_{6}>$ 0 , there exists $T>T_{4}$, such that

$$
z(t) \geq m_{3}\left(\delta_{3}-\varepsilon_{6}\right)=\xi_{3}, \quad \text { for } t>T,
$$

where

$$
\delta_{3}=\frac{1}{m^{M} M_{3}}\left(r_{2}^{L}+\frac{a_{2}^{L} e_{2}^{L} \xi_{1}}{b_{2}^{M}+\xi_{1}}+\frac{a_{3}^{L} e_{3}^{L} \xi_{2}}{b_{3}^{M}+\xi_{2}}\right)
$$

Theorem 6. If conditions (H1)-(H6) hold, then system (1) is permanent.

Proof. If we denote $\xi=\min \left\{\xi_{1}, \xi_{2}, \xi_{3}\right\}, \eta=\max \left\{\eta_{1}, \eta_{2}, \eta_{3}\right\}$. Further, if conditions (H1)-(H6) hold, then any solution $(x(t), y(t), z(t))^{T}$ of system (1) will eventually enter and remain in the compact set $K=\{\xi \leq x, y, z \leq \eta\}$.

That is to say, system (1) is permanent.

Remark 7. From the above theorems, we know that if we choose suitable control conditions $(\mathrm{H} 1)-(\mathrm{H} 6)$, then the system will be controlled to be permanent. These results may provide some reasonable control strategies for relevant ecological departments.

\section{Numerical Simulations and Discussions}

In the above section, we focused our attention on the permanence of the food-chain system with multiple delays in theory; some control conditions have been obtained to guarantee the permanence of system (1). In this section we will give some numerical examples and simulations and then make some discussions. We denote

$$
\begin{aligned}
& f:=r_{1}^{L}-\left(\frac{a_{1}^{M} \eta_{1} \eta_{2}}{b_{2}^{L}+\eta_{1}}+\frac{a_{2}^{M} \eta_{1} \eta_{3}}{b_{2}^{L}+\eta_{1}}\right) ; \\
& g:=r_{2}^{L}+\frac{a_{2}^{L} e_{2}^{L} \xi_{1}}{b_{1}^{M}+\xi_{1}}-\frac{a_{3}^{M} \eta_{3}}{b_{3}^{L}} .
\end{aligned}
$$



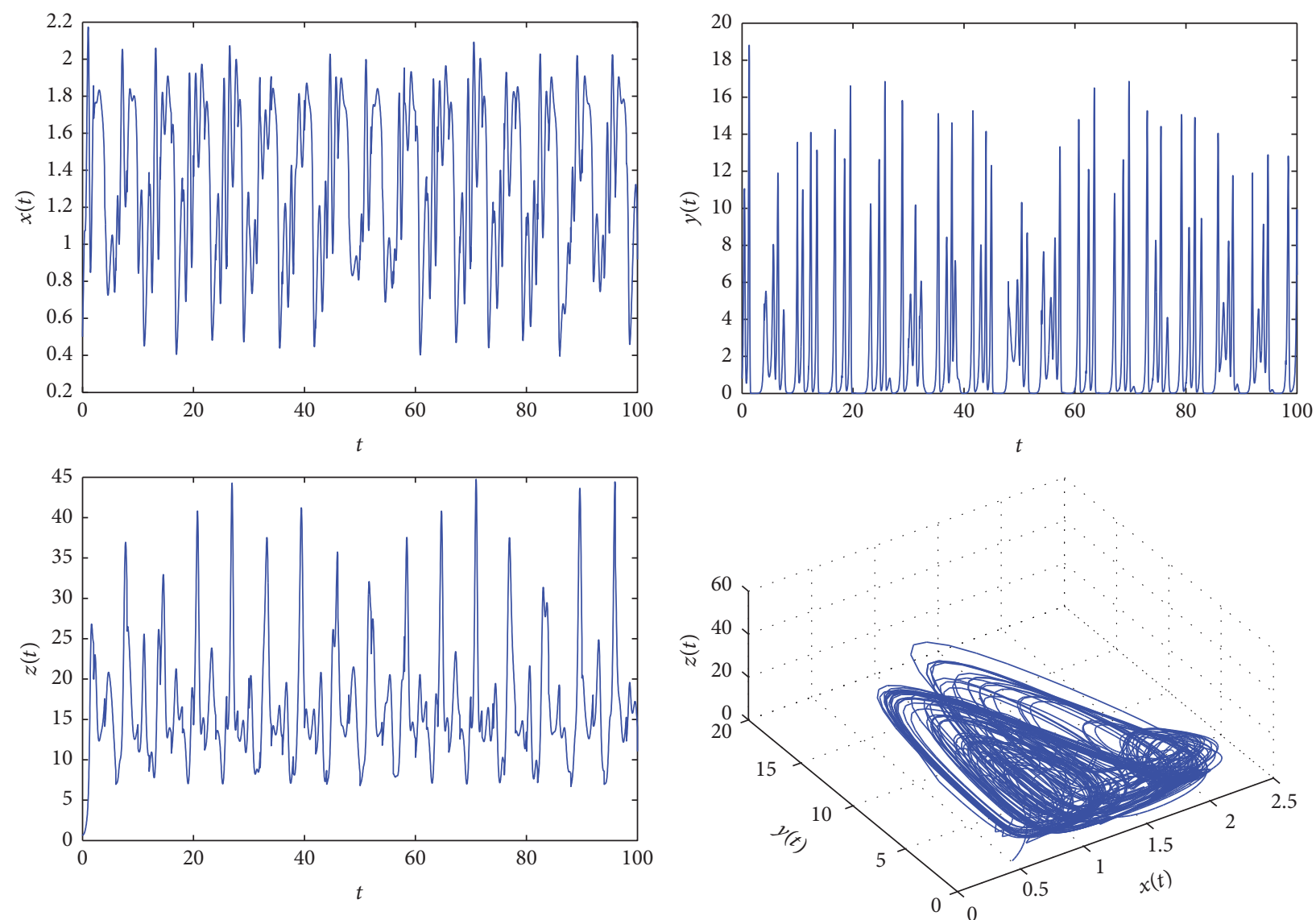

Figure 5: Effect of delays on the dynamical behavior of system (1) with $\phi_{1}(s)=x(0)=0.5, \phi_{2}(s)=y(0)=0.8, \phi_{3}(s)=z(0)=0.6, s \in[-\tau, 0]$.

According to Theorem 5, we can see that the signs of $f$ and $g$ are very important factors for controlling the permanence of system (1). In order to verify this point, we only change one or two parametric values based on Case 1 as follows.

Case 1. We consider the following choice of parametric values:

$$
\begin{aligned}
& r_{1}(t)=4+\sin (t), \\
& r_{2}(t)=b+\cos (t), \\
& k_{1}(t)=2+0.1 \sin (t), \\
& k_{2}(t)=3, \\
& b_{1}(t)=3+\sin (t), \\
& b_{2}(t)=4-0.1 \cos (t), \\
& b_{3}(t)=4, \\
& m(t)=0.2+0.1 \cos (2 t), \\
& a_{1}(t)=1, \\
& a_{2}(t)=0.2, \\
& a_{3}(t)=2, \\
& e_{1}(t)=18,
\end{aligned}
$$

$$
\begin{aligned}
e_{2}(t) & =3, \\
e_{3}(t) & =6, \\
q_{1 k} & =0.1, \\
q_{2 k} & =0.2, \\
q_{3 k} & =0.2,
\end{aligned}
$$

$$
k=1,2, \ldots
$$

with initial conditions

$$
\begin{aligned}
& \phi_{1}(s)=x(0)=0.5, \\
& \phi_{2}(s)=y(0)=0.8, \\
& \phi_{3}(s)=z(0)=0.6,
\end{aligned}
$$

$$
s \in[-\tau, 0] .
$$

The delays are given as

$$
\begin{aligned}
& \tau_{1}=\tau_{2}=\tau_{3}=0.01, \\
& \tau_{4}=\tau_{5}=\tau_{6}=0.02 .
\end{aligned}
$$

On the one hand, one can verify that conditions (H1)(H6) are satisfied. According to Theorem 5, system (1) is permanent. On the other hand, system (1) is numerically solved 
for the above choice of parameters and initial conditions. It is obvious that the system is permanent and has quasiperiodic solutions; it is clear to see the time-series and phase portrait intuitively in Figure 1.

Case 2. If we increase the value of $a_{1}^{M}$, for example, if we let $a_{1}(t)=3.5$ while other parametric values are the same as Case 1 , then system (1) is numerically solved in Figure 2. One can find that the highest predator $z$ will be extinct finally, and the density of the prey will decrease to zero periodically, while the middle predator can be permanent.

Case 3. When we go on increasing the value of $a_{1}^{M}$ such that $a_{1}(t)=3.9$, one can see that both the highest predator $z$ and the prey $x$ will be extinct finally, while the middle predator $y$ can be permanent at the moment (see Figure 3).

Case 4. If we increase the value of $a_{3}^{M}$, for example, we let $a_{3}(t)=2.3$, while other parametric values are the same as Case 1. It is very strange and interesting that both the highest predator $z$ and the prey $x$ can be permanent, while the middle predator $y$ will be extinct gradually (see Figure 4).

Case 5. In the following, we will study effects of the delays on the dynamical behavior of the system. To this end, we enlarge the value of all of the delays and choose

$$
\tau_{1}=\tau_{2}=\tau_{3}=\tau_{4}=\tau_{5}=\tau_{6}=0.2,
$$

while the other parameters and initial conditions are the same as Case 1. It is easy to verify that condition (H4) does not hold any more. By the numerical simulations, we can see that the prey $x$ and the higher predator $z$ will be permanent while the density of the lower predator $y(t)$ oscillates, and it shows a strange dynamic characteristic of intermittent extinction (see Figure 5).

By the above theoretical analysis and the numerical experiments, it is shown that we can seek some reasonable control strategies so that the system can be controlled to be permanent, such as changing values of some important parameters of the system. On the other hand, by the numerical simulations in Case 5, when the delays are too long, the density of the species will oscillate and leads to strange phenomena of intermittent extinction, which can explain the complexity of biological systems. In addition, we can also seek some efficient measures to guarantee that some of the species (beneficial insects) in the system can be permanent while the other species (pest insects) will be extinct finally. And this method can be extended to the study of the other systems with variable coefficients, such as epidemic systems and neural network systems.

\section{Competing Interests}

The authors declare that they have no competing interests.

\section{Acknowledgments}

This work was supported by National Natural Science Foundation of China (11372294 and 41372301), Scientific Research Fund of Sichuan Provincial Education Department (14ZB0115 and 15ZB0113), and Doctorial Research Fund of Southwest University of Science and Technology (15zx7138).

\section{References}

[1] J. Hofbauer and K. Sigmund, Evolutionary Games and Population Dynamics, Cambridge University Press, Cambridge, UK, 1998.

[2] Y. Wang, J. D. Cao, A. Alofi, A. Al-Mazrooei, and A. Elaiw, "Revisiting node-based SIR models in complex networks with degree correlations," Physica A, vol. 437, Article ID 16218, pp. 75-88, 2015.

[3] J. Cao, Y. Wang, A. Alofi, A. Al-Mazrooei, and A. Elaiw, "Global stability of an epidemic model with carrier state in heterogeneous networks," IMA Journal of Applied Mathematics, vol. 80, no. 4, pp. 1025-1048, 2015.

[4] Y. Wang and J. D. Cao, "Global dynamics of multi-group SEI animal disease models with indirect transmission," Chaos, Solitons \& Fractals, vol. 69, pp. 81-89, 2014.

[5] H. Yu, S. Zhong, and R. P. Agarwal, "Mathematics analysis and chaos in an ecological model with an impulsive control strategy," Communications in Nonlinear Science and Numerical Simulation, vol. 16, no. 2, pp. 776-786, 2011.

[6] Y. Shao, B. Dai, and Z. Luo, "The dynamics of an impulsive one-prey multi-predators system with delay and Holling-type II functional response," Applied Mathematics and Computation, vol. 217, no. 6, pp. 2414-2424, 2010.

[7] X. Y. Song and Y. F. Li, "Dynamic complexities of a Holling II two-prey one-predator system with impulsive effect," Chaos, Solitons \& Fractals, vol. 33, no. 2, pp. 463-478, 2007.

[8] Z. Liu and R. Tan, "Impulsive harvesting and stocking in a Monod-Haldane functional response predator-prey system," Chaos, Solitons and Fractals, vol. 34, no. 2, pp. 454-464, 2007.

[9] X. Yang, "Uniform persistence and periodic solutions for a discrete predator-prey system with delays," Journal of Mathematical Analysis and Applications, vol. 316, no. 1, pp. 161-177, 2006.

[10] J. Yan and A. Zhao, "Oscillation and stability of linear impulsive delay differential equations," Journal of Mathematical Analysis and Applications, vol. 227, no. 1, pp. 187-194, 1998.

[11] K. Gopalsamy, Stability and Oscillations in Delay Differential Equations of Population Dynamics, vol. 74 of Mathematics and Its Applications, Kluwer Academic Publishers, Dordrecht, The Netherlands, 1992.

[12] V. Lakshmikantham, D. D. Bainov, and P. S. Simeonov, Theory of Impulsive Differential Equations, vol. 6, World Scientific Publishing, 1989.

[13] V. Kolmanovskii and A. Myshkis, Introduction to the Theory and Applications of Functional Differential Equations, vol. 463 of Mathematics and Its Applications, Kluwer Academic Publishers, Dordrecht, The Netherlands, 1999. 


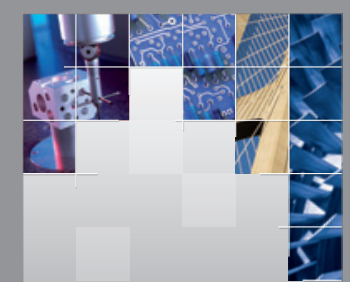

\section{Enfincering}
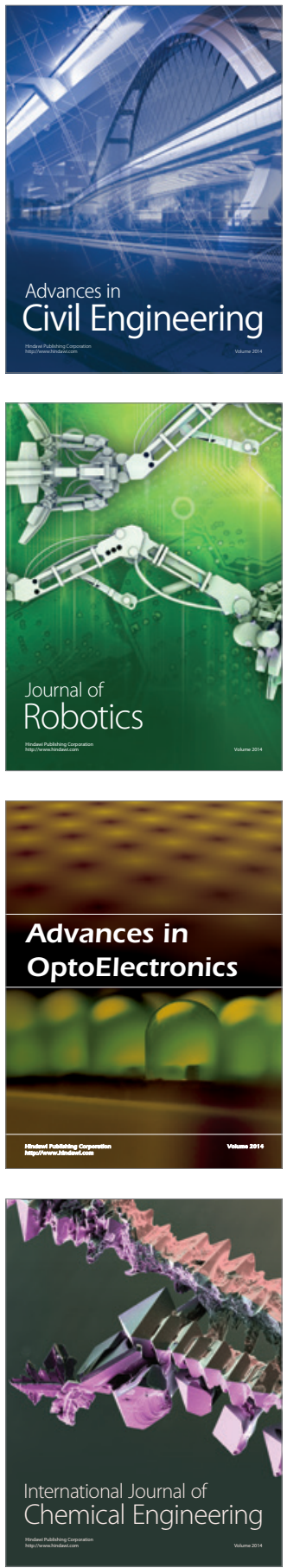

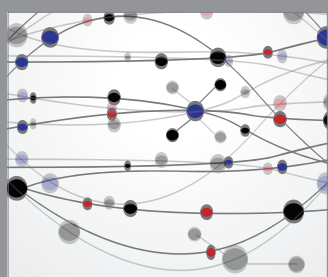

The Scientific World Journal

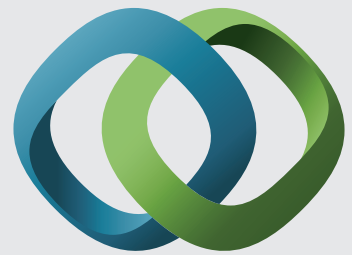

\section{Hindawi}

Submit your manuscripts at

http://www.hindawi.com
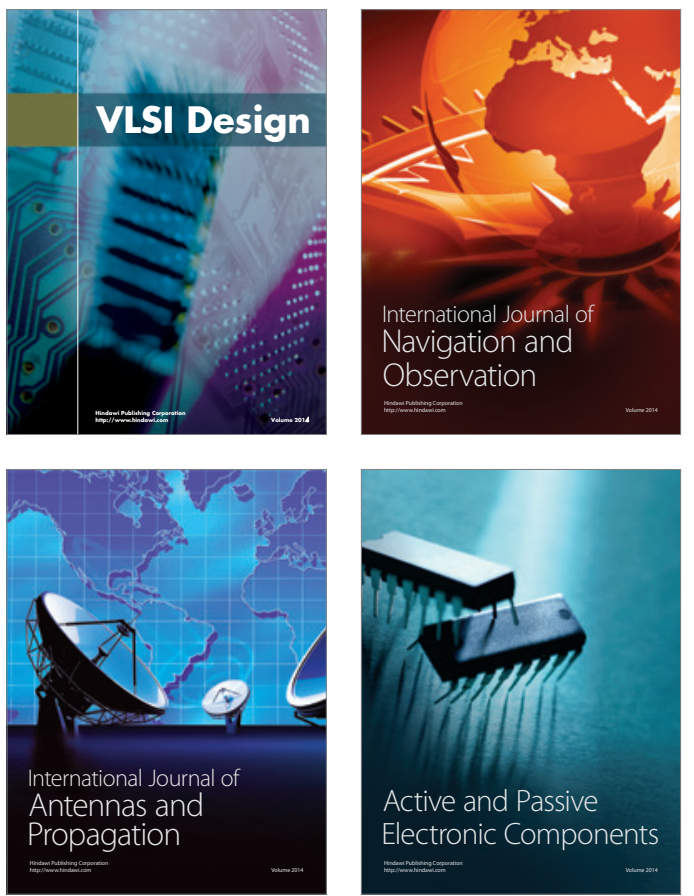
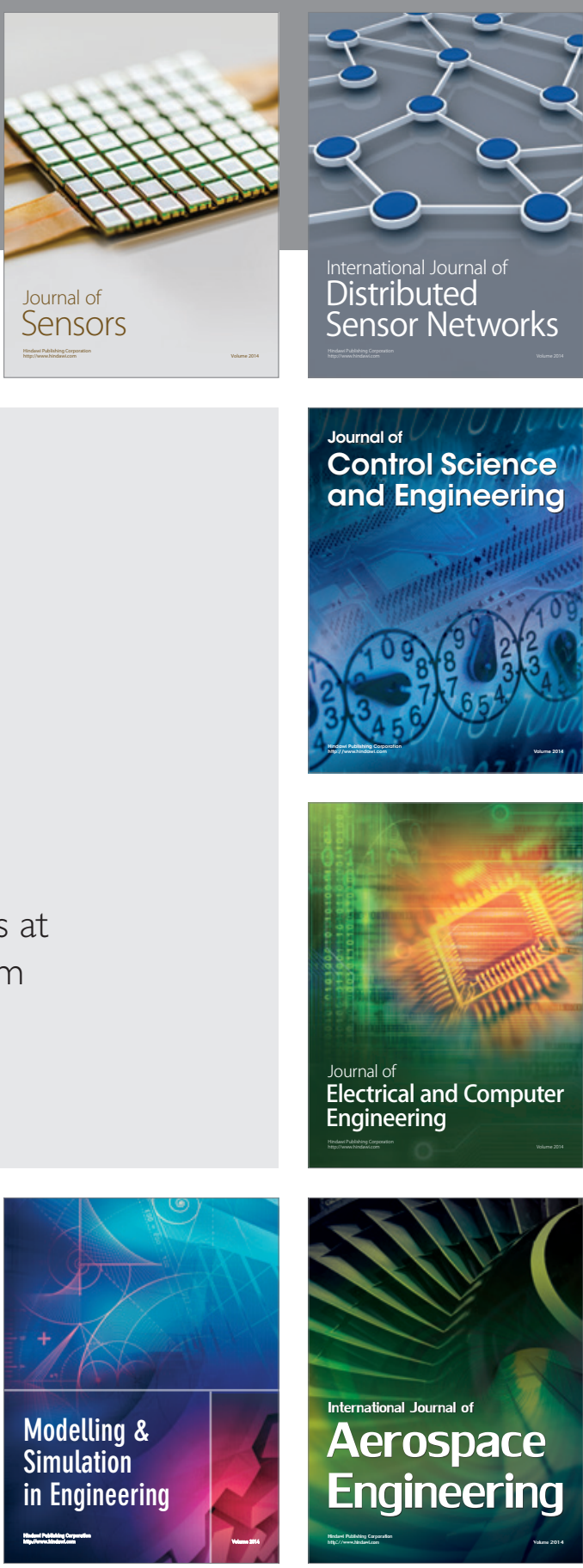

International Journal of

Distributed

Sensor Networks

Journal of

Control Science

and Engineering
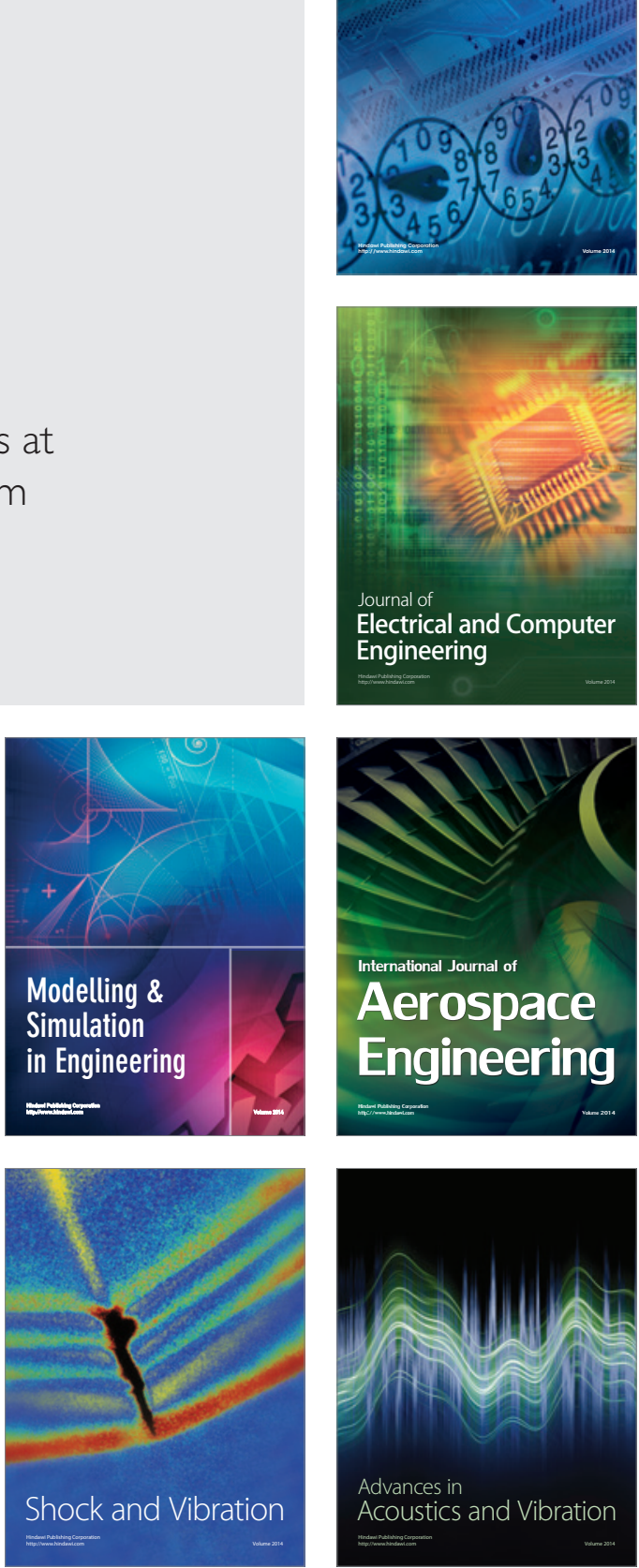